\title{
Synthesis and Surface Activity of Cashew-Based Anion-Nonionic Surfactants
}

\author{
Haiyan $\mathrm{Li}^{1}$, Jun Wang ${ }^{1 *}$, Changhuan Liu ${ }^{1}$, Jun $\mathrm{Han}^{2}$, Cuiqin Li ${ }^{1}$, Mengmeng Ning ${ }^{1}$ \\ ${ }^{1}$ College of Chemistry and Chemical Engineering, Northeast Petroleum University, Daqing, China \\ ${ }^{2}$ Jinzhou Engineering Technology Department of Liaohe Oilfield Company, Jinzhou, China \\ Email: *wangjun1965@yeah.net
}

Received April 13, 2012; revised May 14, 2012; accepted May 23, 2012

\begin{abstract}
Four novel anion-nonionic surfactants were synthesized using cashew phenol as raw material. The four structures were characterized by IR and elemental analysis. Their surface activities were investigated. Their critical micelle concentrations $(C M C)$ are $9.30 \times 10^{-3} \mathrm{~mol} / \mathrm{L}, 8.50 \times 10^{-3} \mathrm{~mol} / \mathrm{L}, 8.10 \times 10^{-3} \mathrm{~mol} / \mathrm{L}$ and $7.71 \times 10^{-3} \mathrm{~mol} / \mathrm{L}$ respectively, and the corresponding surface tensions at CMC are $28.38 \mathrm{mN} / \mathrm{m}, 28.60 \mathrm{mN} / \mathrm{m}, 30.40 \mathrm{mN} / \mathrm{m}$ and $30.00 \mathrm{mN} / \mathrm{m}$ respectively. The contact angles of the solutions on sheet galsses were measured to observe their surface wettabilities. The effects of their concentrations, the concentrations of $\mathrm{NaCl}$ and temperature on their foaming capacity and foam stability were studied.
\end{abstract}

Keywords: Cashew Phenol; Anion-Nonionic Surfactant; Surface Activity; CMC

\section{Introduction}

Anionic surfactants are easy to generate precipitation so as to lose surface activity in high salinity formation water, and nonionic surfactants are difficult to dissolve when temperature exceeds the cloud point. But anion-nonionic surfactants can overcome the weaknesses of the single anionic or nonionic surfactants and apply to the reservoir conditions of high salinity and high temperature [1,2].

Cashew phenol is cheap, green and easy to get in nature, so the surfactants synthesized by cashew phenol have better ecological performance and accord with the demand of "green raw materials" of green surfactants. There are no reports on anion-nonionic surfactants with cashew phenol as starting material till now.

In the present study, we prepared a series of novel surfactants-cashew phenol polyoxyethylene-carboxylates modified anion-nonionic surfactants containing nonionic and anionic hydrophilic moieties and investigated their properties. In this paper, we report the surface activities of theses surfactants, including their surface tension, wetting and foaming properties, and they exhibit surface activities similar to those of traditional surfactants [3].

\section{Materials and Methods}

\subsection{Materials}

Cashew phenol was supplied by Shanghai Meidong Biological Material Co., Ltd. in commercial grade. Ethylene

${ }^{*}$ Corresponding author. oxide was supplied Liaoyang Petrochemical Company in commercial grade. Chloroacetic acid, sodium hydroxide, potassium hydroxide and acetone, purchased from Tianjin Damao chemical reagent factory, were of analysis grade.

\subsection{Preparation of Cashew Phenol Polyoxyethylene-Carboxylates Modified Anion-Nonionic Surfactants}

We obtained novel surfactants possessing anion-nonionic structures through a three-step process (shown in Scheme 1) [4]. In the first step, a series of polyoxyethylene ethers presenting hydrophilic ethylene oxide chain segments at phenolic hydroxyl were obtained through ring-opening polymerizations of cashew phenol with ethylene oxide in the presence of potassium hydroxide as a catalyst. In the second step, these polyoxyethylene ethers and chloroacetic acid were reacted with sodium hydroxide. The third step was nucleophilic substitution reaction. The following description is typical of the procedures used to prepare the anion-nonionic surfactants: cashew phenol $(0.5 \mathrm{~mol})$, ethylene oxide ( $4 \mathrm{~mol}, 5 \mathrm{~mol}, 6 \mathrm{~mol}, 7 \mathrm{~mol}$ respectively), and potassium hydroxide (catalyst, $1 \mathrm{~g}$ ) was stirred mechanically and heated to $120^{\circ} \mathrm{C}-140^{\circ} \mathrm{C}$ under the pressure of $0.25 \pm 0.05 \mathrm{MPa}$. Polyoxyethylene ether $(0.02 \mathrm{~mol}$, $13.04 \mathrm{~g}, 14.8 \mathrm{~g}, 16.56 \mathrm{~g}, 18.32 \mathrm{~g})$, chloroacetic acid (3.78 g), and sodium hydroxide $(6.4 \mathrm{~g})$ was stirred magnetically and heated $30^{\circ} \mathrm{C}$ for $1 \mathrm{~h}$, and then heated $60^{\circ} \mathrm{C}$ for $4.5 \mathrm{~h}$ under the pressure of $5.32 \pm 0.05 \mathrm{KPa}$. The sodium salts of the products were washed with acetone. Compounds A, 
B, C and D possess 8, 10, 12 and 14 ethylene oxide units, respectively (as depicted in Scheme 1).

Step.1: ring-opening polymerization

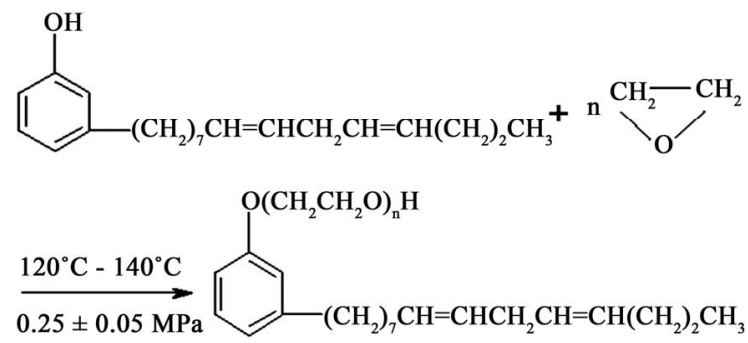

Step.2: alkalization reaction

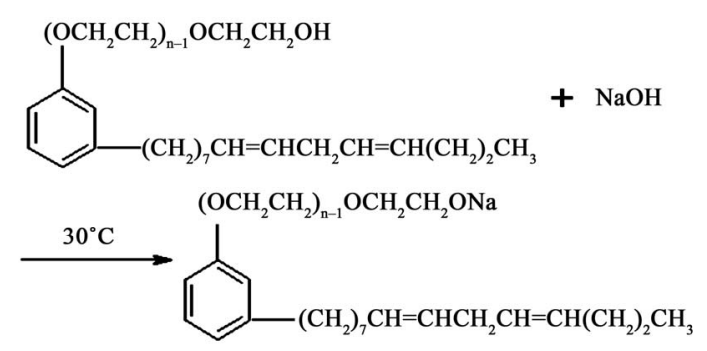

Step.3: nucleophilic substitution reaction

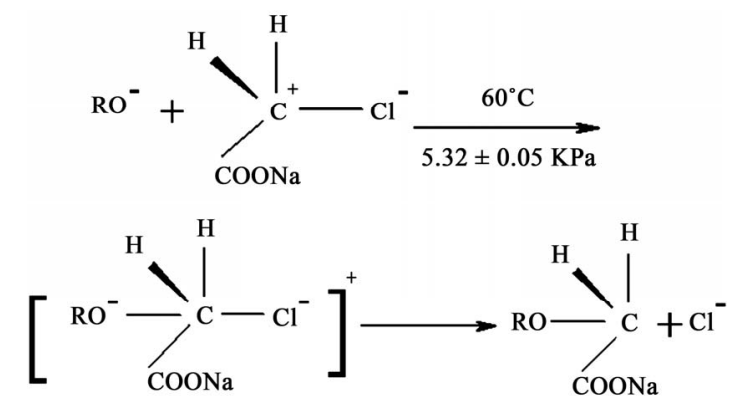

$\mathrm{R}$ :

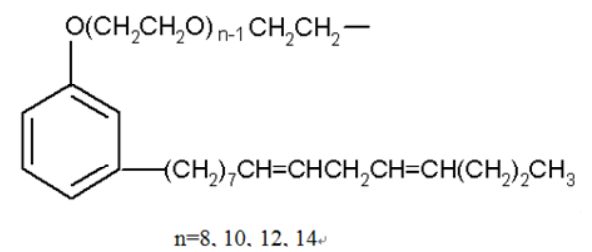

Scheme 1. Synthesis of anion-nonionic surfactants A, B, C and $\mathrm{D}$.

\subsection{Analysis}

The structures of the final products were confirmed through infrared (IR) and elemental analysis. IR spectra recorded in the range $4000-600 \mathrm{~cm}^{-1}$ were obtained using a Japan Spectroscopic FT/IR ${ }^{-3}$ spectrometer. The materials were ground with $\mathrm{KBr}$ and smeared onto pellets. Elemental analysis datas were obtained using a Germany Heraeus Elemental Analyzer.

\subsection{Measurements}

Surface tensions were determined by Hanging Drop Method using a JC2002CI surface tensiometer. and the temperature was maintained precisely at $25^{\circ} \mathrm{C}$. Contact angles were measured using a JC2002CI intravenous drip contact angle meter. The foaming properties were determined using the Ross-Miles method and the temperature was maintained precisely at $45^{\circ} \mathrm{C}$ except the temperature effect. The foaming capacity was measured in terms of the height of the foam produced initially; the foam stability was measured in terms of the height after $5 \mathrm{~min}$.

\section{Results and Discussion}

\subsection{Preparation of Cashew Phenol Polyoxyethylene-Carboxylates Modified Anion-Nonionic Surfactants}

The four structures were confirmed through IR and elemental analysis (the results are shown in Table 1). The IR spectra display bands at 3000-3010 (ph-H), $1715-1760(\mathrm{C}=\mathrm{O}), 1680-1620(-\mathrm{CH}=\mathrm{CH}-), 1340-1380\left(\mathrm{CH}_{3}\right)$, 1210-1275(-O-), 110-1225(- $\left.\mathrm{CH}_{2} \mathrm{CH}_{2} \mathrm{O}-\right), 750-810$ (ph-H), 7 $25-780\left(\mathrm{CH}_{2}\right)[5]$.

\subsection{Surface Tension}

Figure 1 shows plots of the surface tensions vs molar concentrations of anion-nonionic surfactants A, B, C and D. CMC values of each surfactant given according to the intersection points of the extension of the straight part of both sides of turning point in Figure $\mathbf{1}$ are shown in Table $2[6,7]$. An increase in the length of the polyoxyethylene chain of the nonionic portion resulted in a decrease in the surface activity. This phenomenon is related to the increased hydrophilicity decreasing the concentration of the surfactants at the surface [8].

\subsection{Wetting Power}

Surface tension of water is larger in the common liquid and it can not be wetting and spreading on the glass surface, after adding surfactant the surface tension of water

Table 1. Elemental analysis of cashew phenol polyoxyethylenecarboxylates modified anion-nonionic surfactants (F:Found C: Calculated).

\begin{tabular}{ccccccccc}
\hline & & \multicolumn{6}{c}{ Elemental analysis } \\
\cline { 3 - 8 } Compounds & Units of EO & \multicolumn{2}{c}{$\mathrm{C}(\%)$} & \multicolumn{2}{c}{$\mathrm{H}(\%)$} & \multicolumn{2}{c}{$\mathrm{O}(\%)$} \\
\cline { 3 - 8 } & & $\mathrm{F}$ & $\mathrm{C}$ & $\mathrm{F}$ & $\mathrm{C}$ & $\mathrm{F}$ & $\mathrm{C}$ \\
\hline $\mathrm{A}$ & 8 & 63.93 & 64.36 & 8.88 & 9.33 & 24.04 & 24.39 \\
$\mathrm{~B}$ & 10 & 62.39 & 60.15 & 8.90 & 8.72 & 25.37 & 27.69 \\
$\mathrm{C}$ & 12 & 62.11 & 60.79 & 8.92 & 8.89 & 26.43 & 28.15 \\
$\mathrm{D}$ & 14 & 61.45 & 60.84 & 8.94 & 9.02 & 27.31 & 28.69 \\
\hline
\end{tabular}




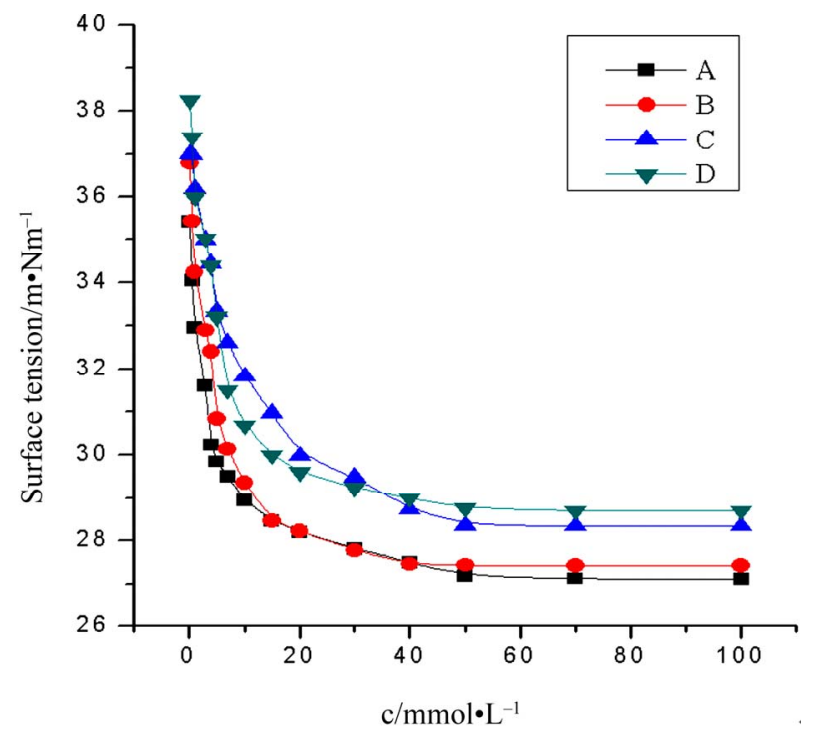

Figure 1. Surface tensions of $A, B, C$ and $D$ in aqueous solution at $25^{\circ} \mathrm{C}$.

Table 2. CMC values and surface tensions of anion-nonionic surfactants at $25^{\circ} \mathrm{C}$.

\begin{tabular}{ccccc}
\hline $\mathrm{R}$ & A & B & C & D \\
\hline $\mathrm{CMC}(\mathrm{mmol} / \mathrm{L})$ & 9.30 & 8.50 & 8.10 & 7.71 \\
$\gamma_{\mathrm{CMC}}(\mathrm{mN} / \mathrm{m})$ & 28.38 & 28.60 & 30.40 & 30.00 \\
\hline
\end{tabular}

can significantly reduce, but also may reduce the solidliquid interfacial tension, so that it can be spontaneous re-spread on the glass surface [9]. Usually strong hydrophilic surfactant has good wetting, and therefore the contact angle can be measured in aqueous solution of surfactant on the glass slide to respond to its wettability.

Figure 2 shows the contact angles formed beween surfactant solutions and glass slide vs concentrations of the four surfactants. The smaller contact angles observed for the solutions containing the surfactants, compared with that of water alone, indicate that these compounds possess wetting power on the glass slide. With their concentrations increased, the contact angles decreased. When their concentrations exceeded $5000 \mathrm{mg} / \mathrm{L}$, they almost kept the values and behaved the most effective wet ability.

\subsection{Foaming Properties}

Tables 3-6 lists the foam properties of four anion-nonionic surfactants. Table 3 indicated that with the concentrations of the four products increased, the foaming capacity (measured in terms of the height of foam initially produced) and foam stability (measured in terms of the height after $5 \mathrm{~min}$ ) of them increased. After reaching a certain degree, they kept the value all long [10-12]. When adsorbing in the gas-liquid interface, the hydration of the hydrophilic EO chain in molecules makes the hy- drophilic groups of the surface form the inter-molecular hydrogen bonding or cross-cut in order to wind each other so that surface film has a certain strength, so the products have a foaming ability and foam stability. With the increase of their concentrations, the concentration of hydrophilic EO groups increased, the foaming capacity and foam stability gradually increased, but when the concentration reached a certain degree, the hydrophobic of EO chain started to pick up, so foaming capacity and foam stability were almost no change.

Table 4 indicated that with temperature increased, the foaming capacity and foam stability decreased. This was mainly due to the increase of temperature, liquid viscosity and surface viscosity dropped and the foam evaporation rate and inter-bubble gas diffusion rate increased, and therefore the decay process of foam accelerated, so the foaming capacity and foam stability decreased.

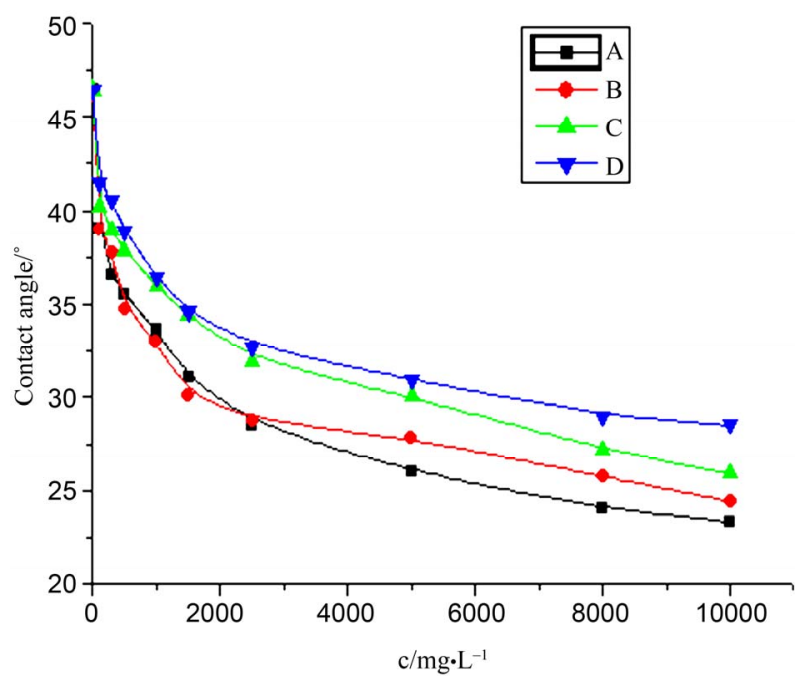

Figure 2. Contact angles of $A, B, C$ and $D$ in aqueous solution at $25^{\circ} \mathrm{C}$.

Table 3. The effect of the concentrations of the four surfactants on their foaming properties.

\begin{tabular}{ccccccc}
\hline $\mathrm{C}_{\mathrm{gL}}$ & 3 & 4 & 5 & 6 & 7 & 8 \\
\hline $\mathrm{H}_{0 \min } / \mathrm{cm}$ & 10.5 & 10.6 & 10.8 & 11.0 & 11.3 & 11.3 \\
$\mathrm{H}_{5 \min } / \mathrm{cm}$ & 8.0 & 8.3 & 8.5 & 9.0 & 9.0 & 9.0 \\
$\mathrm{H}_{0 \min } / \mathrm{cm}$ & 12.5 & 12.8 & 13.0 & 13.8 & 14.0 & 13.8 \\
$\mathrm{H}_{5 \min } / \mathrm{cm}$ & 11.0 & 11.5 & 11.8 & 12.0 & 12.0 & 12.1 \\
$\mathrm{H}_{0 \min } / \mathrm{cm}$ & 13.0 & 13.3 & 13.5 & 13.8 & 14.0 & 14.0 \\
$\mathrm{H}_{5 \min } / \mathrm{cm}$ & 12.0 & 12.3 & 12.8 & 12.9 & 13.0 & 13.0 \\
$\mathrm{H}_{0 \min } / \mathrm{cm}$ & 12.0 & 13.8 & 14.3 & 14.3 & 14.3 & 14.3 \\
$\mathrm{H}_{5 \min } / \mathrm{cm}$ & 10.3 & 11.8 & 12.5 & 12.8 & 12.5 & 12.5 \\
\hline
\end{tabular}


Table 5 indicated that with the concentrations of $\mathrm{NaCl}$ increased, the foaming capacity and foam stability increased at first and then decreased. This was mainly because on the one hand, after adding electrolyte, ionic atmosphere and the thickness of diffuse double layer of the surfactant ionomers were compressed, and therefore decreased the repulsion between them, so that surfactant ions more quickly adsorbed on the surface to form micelles; and with the $\mathrm{Na}^{+}$concentration increased, more $\mathrm{Na}^{+}$into the ionic fog, micelles and adsorption layers, the formation of the surface micelles speeded up, so that $\gamma$, cmc decreased. In general, the lower $\gamma$ and the smaller $\mathrm{cmc}$, the more easily bubble and the more stable foam. On the other hand, $\mathrm{NaCl}$ reached a certain concentration, diffuse double layer of the membrane was compressed, and reduced repulsion between the membrane and accelerated the discharge fluid process. Therefore, $\mathrm{NaCl}$ addition of a small amount, will help improve the foam performance of the products.

Table 6 indicated that with the concentrations of $\mathrm{Ca}^{2+}$, $\mathrm{Mg}^{2+}$ increased, the foaming capacity and foam stability

Table 4. The effect of temperature on foaming properties of the four surfactants.

\begin{tabular}{ccccccc}
\hline \multicolumn{2}{c}{ Temperature $/{ }^{\circ} \mathrm{C}$} & 25 & 35 & 45 & 55 & 65 \\
\hline $\mathrm{A}$ & $\mathrm{H}_{0 \min } / \mathrm{cm}$ & 11 & 10.7 & 10.6 & 10.1 & 10 \\
& $\mathrm{H}_{5 \min } / \mathrm{cm}$ & 8.6 & 8.5 & 8.3 & 7.5 & 6.4 \\
$\mathrm{~B}$ & $\mathrm{H}_{0 \min } / \mathrm{cm}$ & 12.8 & 12.6 & 12.5 & 12 & 11.8 \\
& $\mathrm{H}_{5 \min } / \mathrm{cm}$ & 11.3 & 11.1 & 11.0 & 9.8 & 7.6 \\
$\mathrm{C}$ & $\mathrm{H}_{0 \min } / \mathrm{cm}$ & 13.3 & 13.4 & 13.0 & 12 & 11.1 \\
& $\mathrm{H}_{5 \min } / \mathrm{cm}$ & 12.5 & 12.5 & 12.0 & 9.9 & 8.3 \\
\multirow{2}{*}{$\mathrm{D}$} & $\mathrm{H}_{0 \min } / \mathrm{cm}$ & 14.3 & 13.9 & 13.8 & 13.3 & 12.7 \\
& $\mathrm{H}_{5 \min } / \mathrm{cm}$ & 11.9 & 11.8 & 11.3 & 10.7 & 8.1 \\
\hline
\end{tabular}

Table 5. The effect of the concentrations of $\mathrm{NaCl}$ on foaming properties of the four surfactants.

\begin{tabular}{ccccccccc}
\hline $\mathrm{W}(\mathrm{NaCl}) / \%$ & 0 & 0.5 & 1.0 & 2.0 & 3.0 & 4.0 & 5.0 \\
\hline $\mathrm{A}$ & $\mathrm{H}_{0 \mathrm{~min}} / \mathrm{cm}$ & 10.6 & 11.5 & 12.3 & 12.3 & 11.9 & 10.3 & 5.9 \\
& $\mathrm{H}_{5 \mathrm{~min}} / \mathrm{cm}$ & 8.3 & 9.0 & 10.0 & 9.6 & 9.5 & 7.0 & 3.0 \\
$\mathrm{~B}$ & $\mathrm{H}_{0 \mathrm{~min}} / \mathrm{cm}$ & 12.5 & 12.5 & 13.5 & 13.2 & 13.0 & 12.3 & 12.0 \\
& $\mathrm{H}_{5 \mathrm{~min}} / \mathrm{cm}$ & 11.0 & 11.0 & 11.4 & 11.3 & 11.0 & 10.3 & 9.5 \\
$\mathrm{C}$ & $\mathrm{H}_{0 \mathrm{~min}} / \mathrm{cm}$ & 13.0 & 13.5 & 12.3 & 11.9 & 11.9 & 11.8 & 11.3 \\
& $\mathrm{H}_{5 \mathrm{~min}} / \mathrm{cm}$ & 12.0 & 12.2 & 8.8 & 8.8 & 8.7 & 8.5 & 7.8 \\
& $\mathrm{H}_{0 \mathrm{~min}} / \mathrm{cm}$ & 13.8 & 14.0 & 11.8 & 11.5 & 11.3 & 10.8 & 10.6 \\
& & & & & & & & \\
& $\mathrm{H}_{5 \mathrm{~min}} / \mathrm{cm}$ & 11.3 & 11.5 & 5.5 & 5.5 & 5.5 & 4.5 & 4.3 \\
\hline
\end{tabular}

Table 6. The effect of the concentrations of $\mathrm{Ca}^{2+}, \mathrm{Mg}^{2+}$ on foaming properties of the four surfactants.

\begin{tabular}{ccccccccc}
\hline \multirow{2}{*}{$\mathrm{w}\left(\mathrm{Ca}^{2+}, \mathrm{Mg}^{2+}\right) / 10^{-6}$} & 0 & 0.5 & 1.0 & 2.0 & 3.0 & 4.0 & 5.0 \\
\hline \multirow{2}{*}{$\mathrm{A}$} & $\mathrm{H}_{0 \min } / \mathrm{cm}$ & 10.6 & 10.6 & 10.5 & 10.4 & 10.1 & 9.8 & 9.5 \\
& $\mathrm{H}_{5 \min } / \mathrm{cm}$ & 8.3 & 8.3 & 8.1 & 8.0 & 7.9 & 7.9 & 7.8 \\
\multirow{2}{*}{$\mathrm{B}$} & $\mathrm{H}_{0 \min } / \mathrm{cm}$ & 12.5 & 12.5 & 12.3 & 12.3 & 12.2 & 12.0 & 11.5 \\
& $\mathrm{H}_{5 \min } / \mathrm{cm}$ & 11.0 & 11.0 & 10.3 & 9.5 & 9.5 & 9.5 & 9.0 \\
\multirow{2}{*}{$\mathrm{C}$} & $\mathrm{H}_{0 \min } / \mathrm{cm}$ & 13.0 & 12.3 & 12.2 & 11.9 & 11.8 & 11.6 & 10.9 \\
& $\mathrm{H}_{5 \min } / \mathrm{cm}$ & 12.0 & 10.9 & 10.7 & 10.7 & 10.4 & 10 & 8.8 \\
\multirow{2}{*}{$\mathrm{D}$} & $\mathrm{H}_{0 \min } / \mathrm{cm}$ & 13.8 & 12.9 & 12.5 & 12.2 & 11.5 & 11.2 & 10.8 \\
& $\mathrm{H}_{5 \min } / \mathrm{cm}$ & 11.3 & 10.7 & 10.7 & 10.6 & 10.5 & 10.3 & 9.3 \\
\hline
\end{tabular}

decreased. This was because, from the molecular structure $-\mathrm{CH}_{2} \mathrm{COO}$ - possessed poor resistance to hard water, when encountering $\mathrm{Ca}^{2+}, \mathrm{Mg}^{2+}$, easily generated curdlike material, and therefore affected the foam properties.

\section{Conclusion}

We prepared a series of novel cashew phenol polyoxyethylene-carboxylates modified anion-nonionic surfactants through ring-opening polymerization, alkalizetion reaction and nucleophilic substitution reaction. Because of the unique structural features resulting from the presence of ethylene oxide and carboxylic hydrophilic groups in a single molecule, the auxiliaries exhibit good surface activities, including low-surface tension, well-foaming and wetting.

\section{REFERENCES}

[1] A. M. Al-Ghamdi and H. A. Nasr-El-Din, "Effect of Oilfield Chemicals on the cloud Point of Nonionic Surfactants," Colloids and Surfaces A: Physicochemical and Engineering Aspects, Vol. 125, No.1, 1997, pp. 5-18. doi:10.1080/028418501127346846

[2] Y. F. Wang, L. S. Wang, J. Y. Li and F. L. Zhao, "Surfactants Oil Displacement System in High Salinity Formations: Research and Application," Society of Petroleum Engineers (SPE), Allen, 2001, pp. 330-336.

[3] H. J. Liu, L. H. Lin and K. M. Chen, "Reparation and Properties of water-Soluble Polyester Surfactants. II. Preparation and Surface Activity of Silicone-Modified Polyester Surfactants," Journal Applied Polymer Science, Vol. 86, No. 12, 2002, pp. 3005-3012. doi:10.1002/app.11290

[4] Y. Fujita and M. Reinhard, "Identification of Metabolites from the Biologoval Transformation of the Nonionic Surfactant Residue Octyphenoxyacetic Acid and Its Brominated Analog," International Journal of Environmental Science and Technology, Vol. 31, No. 5, 1997, pp. 15181524. doi:10.1021/es9607852

[5] X. Xu, H. Chen and X. R. Cai, "Synthesis and Properties of Polyfluorene Copolymers Bearing Thiophene and Porphyrin," Journal of Chinese Chemical Letters, Vol. 18, 
No. 7, 2007, pp. 879-882. doi:10.1016/j.cclet.2007.05.040

[6] C. C. Lai and K. M. Chen, "Preparation and surface Activity of Polyoxyethylene-Carboxylated Modified Gemini Surfactants," Journal of Colloids and Surfaces A: Physicochemical and Engineering Aspects, Vol. 320, No.1-3, 2008, pp. 6-10. doi:10.1016/j.colsurfa.2007.12.056

[7] L. G. Qiu, A. J. Xie and Y. H. Shen, "Synthesis and Surface Activity of Novel Triazole-Based Cationic Gemini Surfactants," Journal of Chinese Chemical Letters, Vol. 14, No. 6, 2003, pp. 653-656.

[8] M. J. Rosen, "Wetting and Its Modification by Surfactants," Surfactants and Interfacial Phenomena, 3rd Edition, WileyInterscience, New York, 1978, p. 174.

[9] S. Paria and K. C. Khilar, "A Review on Experimental Studies of Surfactant Adsorption at the Hydrophilic SolidWater Interface," Advances in Colloid and Interface Science, Vol. 110, No. 3, 2004, pp. 75-95. doi:10.1016/j.cis.2004.03.001

[10] K. M. Chen and H. J. Liu, "Preparation and Surface Activity of Water-Soluble Polyesters," Journal Applied Polymer Science, Vol. 34, No. 5, 1987, pp. 1879-1888. doi:10.1002/app.1987.070340507

[11] Y. Li, P. Zhang, G. Q. Zhao, X. L. Cao, Q. W. Wang and H. Y. Wang. "Effect of Equilibrium and Dynamic Surface Activity of Surfactant on Foam Transport in Porous Medium," Journal of Colloids and Surfaces A: Physicochemical and Engineering Aspects, Vol. 272, No. 1-2, 2006, pp.124-129. doi:10.1016/j.colsurfa.2005.07.037

[12] D. Beneventi, B. Carre and A. Gandini, "Role of Surfactant Structure on Surface and foaming Properties," Journal of Colloids and Surfaces A: Physicochemical and Engineering Aspects, Vol. 189, No. 1-3, 2001, pp. 65-73. doi:10.1016/S0927-7757(01)00602-1 